\title{
MANAGEMENT OF SUPRACONDYLAR FRACTURE HUMERUS WITH Pink Pulseless Hand in Children.
}

\author{
Mohamed Atea, Ashraf Marzok, Ahmad Addosooki and Mohamed Abdel-Wanis \\ Orthopedics department, Sohag University ,Sohag, Egypt.
}

\begin{abstract}
The management of children with a pink pulseless hand in severely displaced supracondylar humeral fractures remains controversial regarding immediate exploration of the brachial artery and revascularization during fracture fixation or just closed reduction of the fracture and percutaneous pinning and follow up of limb perfusion.Between 2012 and 2016 we followed 52 children with displaced supracondylar fracture humerus. All patients had absent radial pulse with an otherwise well perfused hand. The radial pulse was returned in all patients, without surgical exploration after closed reduction of the fracture and percutaneous pinning .It takes variable periods from 1 week to 2 months . Radial pulse recovery may be due to recanalization of brachial artery or by collaterals. So closed reduction of the fracture, percutaneous pinning and observation is a good option of treatment pediatric supracondylar humeral fractures with a pink pulseless hand without need to do early revascularization procedures.
\end{abstract}

\section{Introduction:-}

Supracondylar fracture humerus is a common injury in children,comprising about $17 \%$ of all pediatric fractures and $62 \%$ of pediatric fractures around the elbow $\{1\}$

.It is common in males, especially between the age of 5 and 8 years $\{2\}$.It results from fall on outstretched hand forcing the humeral distal metaphysis into extension.Flexion type fracture rarely occurs due to trauma to a flexed elbow.Due to the close proximity of vascular structures to the elbow joint, these fractures can be complicated by primary or secondary vascular injury. The incidence of vascular complications presenting about $10 \%\{3\}$ of pediatric supracondylar fracture of humerus. Brachial artery injury can be caused by kinking,spasm,entrapment,Intimal tear ,thrombus formation, partial or complete cut of the artery, on the other hand nerve injuries represent $12 \%$ $20 \%\{4,5\}$ and in most cases it is neuropraxia that resolve spontaneously $\{6,4,7\}$. The treatment of supracondylar fracture humerus with impalpable pulse, white and cold ischaemic hand is immediate reduction of the fracture and exploration of brachial artery if there is no improvement but it is still controversial in patients with pulseless warm hands.. $\{20,21,22\}$ The aim of this study is to address a suitable management plan in children with supracondylar humerus fracture with pulseless but a warm well perfused hand after fracture reduction and fixation.

$\begin{array}{ccl}\text { Patients } & \text { and } & \text { methods:- } \\ \text { It is a prospective } & \begin{array}{l}\text { study of } \\ \text { displaced }\end{array} \\ \text { children } & \text { with } & \text { dish }\end{array}$


supracondylar fracture with pink pulseless hand admitted to Orthopedic Department ,Sohag University Hospital to evaluate the management and fate of cases with warm pink pulseless hand in supracondylar humeral fracture in children. An informed consent had been taken from child's fathers or near relatives and approved from the institute of ethical committee in the period between June 2012 and May 2016. The inclusion criteria were:1) Children from 2-12 years with Supracondylar humeral fracture with warm pink pulseless hand. The exclusion criteria were:1) Pale cold pulseless hand. 2) Patients with immediate postoperative pulse recovery.

3) Open fractures more than grade1.

4) Patients who show postoperative progressive elbow swelling. All children with suspected supracondylar fracture humerus were seen at the orthopedic emergency room .They were assessed generally for other associated injuries and then assessed for the vascular and neurological status of the affected limb. Anteroposterior and lateral radiographs of the elbow were done for all patients. The injured elbow was splinted with elbow in 120 degree of extension . Preoperative investigations (blood picture and prothrombin time vs concentration and arterial duplex) were done for all cases in our study. In our study there were 52 child with Supracondylar fractures of the humerus with well-perfused hand but absent radial pulse, mean age was 4.2 years (range 2-10 years) . 31 patients $(60 \%)$ were boys and 21 patients
$(40 \%)$ were girls .33 patients $(63,3 \%)$ had the fracture on Rt elbow while it was on the left elbow in19 patients $(36,7 \%)$.According to Gartland classifications all patients had type III fractures except 3 patients had type II fractures $(0.05 \%)$. (you need a table of the preoperative date here). General anesthesia was used for all patients with the injured upper limb at the side of the table. The injured elbow was placed on the plate of image intensifier which was adequate for the surgery due to the small size of the elbow. The assistant cleaned and draped the limb along with image intensifier and the fracture was reduced and fixed under image intensifier using 2 or 3 lateral pins then the pulse is checked up .If there was no return we searched for viability, warmness, color of hand and capillary refilling. If the hand was warm, pink with good capillary filling as well as oxygen saturation by oximeter the elbow was immobilized with above elbow posterior slab in 120 degree of extension. All cases with pulse return directly after operation were discarded from study.

Patients had undergone careful continuous supervision in orthopedic department for 5 days postoperatively for any signs of peripheral ischaemia .Warmness, capillary refilling were continually checked. If they were good,the patient was discharged from the hospital for weekly follow up .The $\mathrm{k}$-wires were removed after 4 weeks and the pulse was followed up till its return. Schedule for follow up:1. Immediately post operative. 2. Daily for 5 days post operative, then 
3. One week ,2 week,3 week,4 weeks postoperatively.

4. 1.5 month, 2 month , 3 month and 6 month.

Methods of follow up:Clinically:-

\section{Results:-}

The radial pulse was returned in all patients after varying periods ranging from 1 week to 2 months after closed reduction and fixation of the fracture. In four patients pulse returned after 1 week,in another 10 patients pulse returned after 2 weeks and other 6 cases after 3 weeks, 9 cases after 1month , 20 cases after 1,5 month and 3 cases after 2 month. Male Female Number and time of pulse return postoperative Number Number 4 10 6 9 20 3

\section{We did not explore cubital fossa except in 0}

1. Neurological And vascular assessment.

2. Range of motion. 3. deformity and stiffness. Arterial duplex every two weeks and plain x-ray at 3 weeks and then every 2 weeks till union .

We did not explore cubital fossa except in one case that showed progressive swelling at elbow.We found partial cut in brachial artery and this case was excluded from the study. Five patient with associated median nerve injury was recovered within 6 weeks (mean $4.2+\mathrm{SD}$ ). one patient had radial and another one has ulnar nerve injury. patient with radial nerve palsy spontaneously recovered within 3 months, ulnar nerve recovered after 1,5 month.

The initial reduction was considered to be adequate in most cases. All fractures united at a mean of 3.7 weeks (range; 3.4 to 5 weeks). Cubitus Varus deformity of $7^{\circ}$ relative to the other side was seen in one patient.Painless full range of motion was achieved in all of our patients. Moreover, all patients had the same forearm length and full extension and pronation-supination. During the follow-up, the vascular status of these patients was considered to be satisfactory, as assessed by physical examination including color,temperature,capillary refill and Doppler sonography at the hand and microsurgery outpatient clinic relative to the other normal side.There is no cold intolerance, limb length discrepancy or thrombus formation in the patients under study along follow up periods of 1 to 5 years.

\section{Discussion:-}

Supracondylar fractures of the humerus in children can be classified according to mechanism of fracture into extension type, which represent $97.5 \%$ of all cases, and flexion type, representing the remaining $2.5 \% \quad\{8\}$. Supracondylar fractures of the humerus in children should be considered as a surgical emergency, especially Gartland type III fractures associated with absent radial pulse and cold ischemic hand. Surgical exploration of the cubital fossa is mandatory in patients with absent radial pulses and cold, white hand $\{11$, 
12\}. In such cases, it may be unnecessary to do a lot of imaging studies instead it is better to do urgent exploration.

In this study children with absent radial pulse but with good peripheral circulation were treated by closed reduction and percutaneous pinning. meticulous continuous observation for 5 days in the hospital, then discharged and followed up in outpatient clinic. In all patients radial pulse recovered. Some of the cases showed canalization of the artery and others the collaterals ISSN: 2320-5407 Int. J. Adv. Res. 5(8), 19911995

1994become sufficient with varying periods to recover radial pulse. There is no cold intolerance, limb length discrepancy or thrombus formation in the patients under study along follow up periods of 1 to 5 years. According to the literature, several options have been proposed for the treatment of a pulseless but pink and warm hand, Observation is the treatment of choice for many authors $\{11,13,14\}$. If the hand remains pulseless but well perfused after stabilization, they suggest that the brachial should not be explored and instead rely on collateral circulation. A time window ranging from 12 to $24 \mathrm{~h}$ is usually given in order to rule out vascular spasm. Absence of the radial pulse after the first 24 hours observation indicates that the brachial artery is unlikely to recover patency. According to the same authors, the rich collateral circulation around the elbow is sufficient for the viability of the arm, whereas early revascularization procedures are associated with a high rate of asymptomatic reocclusion and residual stenosis of the brachial artery $\{14\}$. However, the possibility of limb length discrepancy $\{14,15\}$,claudication $\{16$,
$15\}$, cold intolerance $\{17\}$ and thrombus migration $\{18\}$ should be considered if this method of treatment is selected. Radiographic evaluation of the vascular lesion includes both invasive and noninvasive techniques. Doppler, magnetic resonance angiography and colour-flow duplex scanning are non-invasive techniques that may obtain anatomically and hemodynamically useful information.It is our opinion that Doppler sonography is a good, noninvasive tool for follow up to detect amount of blood flow in radial and ulnar.Nerve injuries associated with supracondylar fractures in children occur due to tenting, contusion or entrapment of the nerve within the humeral fragments $\{19\}$. These injuries usually recover spontaneously and have a good prognosis.

Over the 5 years follow up, all the 52 children had recovered the radial pulse. Some recovered by recanalization of the brachial artery confirmed by Doppler study. Others by growth of the collaterals to recover the radial pulse (what is the percentage of each).

\section{Conclusion:-}

Children with supracondylar fractures of the humerus with pulseless, cold ischemic hand needs urgent exploration.Children with pink, warm hand with good capillary refill, stabilization by $\mathrm{k}$,wires and observation is a good option of treatment without need to do early revascularization procedures that can be associated with a high rate of asymptomatic reocclusion and residual stenosis of the brachial artery exploration .

\section{References:-}

1. Noaman HH, . Microsurgical reconstruction of brachial artery injuries in displaced supracondylar fracture humerus in children. J Microsurgery 2006 .26:498-505 2. 2.Lee YH, Lee SK, Kim BS, Chun MS, Baek GH, Gong HS. Three lateral divergent 
or parallel pin fixations for the treatment of displaced supracondylar humerus fractures in children. J Pediatr Orthop 2008;28:417422.

3. Schoenecker PL, Delgado E, Rotman M, Sicard GA, Capelli AM(1996) Pulseless arm in association with totally displaced supracondylar fracture. J Orthop Trauma 10:410-415

4. Campbell CC, Waters PM, Emans JB, Kasser JR, Millis MB (1995) Neurovascular injury and displacement in type III supracondylar humerus fractures. J Pediatr Orthop 15:47-52 toward a goal of prevention. J Pediatr Orthop 16:99-103 5. Culp RW, Osterman AL, Davidson RS, Skirven T, Bora FW Jr(1990) Neural injuries associated with supracondylar fractures of the humerus in children. J Bone Joint Surg Am 72:1211-1215 6. Brown IC, Zinar DM (1995) Traumatic and iatrogenic neurological complications after supracondylar humerus fractures in children. J Pediatr Orthop 15:440-443 7. Ristic S, Strauch RJ, Rosenwasser MP (2000) The assessment and treatment of nerve dysfunction after trauma around the elbow. Clin Orthop Relat Res 370:138-153 8. Wilkins (1996) Fractures and dislocations of the elbow region. In: Rockwood CA, Wilkins KE, King RE (eds) Fractures in children, vol 3, 4th edn. Lippincott-Raven, Philadelphia, pp 363-575 9. Felsenreich F (1931) Kindlichesuprakondylarefrakturen und posttraumatische deformitäten des ellebo engelenk.

10. Gartland JJ (1959) Management of supracondylar fractures of the humerus in children. Surg Gynecol Obstet 109:145-154 11. Garbuz DS, Leitch K, Wright LG (1996) The treatment of supracondylar fractures in children with an absent radial pulse. J Pediatr Orthop 16:594-596 12. Gosens T, Bongers KJ (2003) Neurovascular complications and functional outcome in displaced supracondylar fractures of the humerus in children. Injury 34:267-273

13. Malviya A, Simmons D, Vallamshetla R,
Bache CE (2006) Pink pulseless hand following supracondylar fractures: an audit of British practice. J Pediatr Orthop B 15:62-64

14. Sabharwal S, Tredwell SJ, Beauchamp RD, Mackenzie WG, Jakubec DM, Cairns R, LeBlanc JG (1997) Management of pulseless pink hand in pediatric supracondylar fractures of humerus. J Pediatr Orthop 17:303-310 15. Shaw BA, Kasser JR, Emans JB, Rand FF (1990) Management of vascular injuries in displaced supracondylar humerus fractures without arteriography. J Orthop Trauma 4:25-29 16. Pirone AM, Graham HK, Krajbich JI (1988) Management of displaced extensiontype supracondylar fractures of the humerus in children. J Bone Joint Surg Am 70:641650

17. Marck KW, Kooijman AM, Binnendijk B (1986) Brachial artery rupture following supracondylar fracture of the humerus. Neth J Surg 38:81-84 18. Broudy S, Jupiter J, May JW Jr (1979) Management of supracondylar fracture with brachial artery thrombosis in a child: case report and literature review. J Orthop Trauma 19:540-554 19. Ramachandran M, Birch R, Eastwood DM (2006) Clinical outcome of nerve of injuries associated with supracondylar fractures the humerus in children: the experience of a specialist referral centre. $\mathrm{J}$ Bone Joint Surg $\mathrm{Br}$ 88:90-94 20. Garbuz DS, Leitch K, Wright LG. The treatment of supracondylar fractures in children with an absent radial pulse. J Pediatr Orthop. 1996;16:594-596. 21. Malviya A, Simmons D, Vallamshetla R, Bache CE. Pink pulseless hand following supracondylar fractures: an audit of British practice. J Pediatr Orthop B. 2006;15:62-64. 22. Sabharwal S, Tredwell SJ, Beauchamp RD, Mackenzie WG, Jakubec DM, Cairns $\mathrm{R}$, LeBlanc JG. Management of pulseless pink hand in pediatric supracondylar fractures of humerus. J Pediatr Orthop. 1997;17:303-310. doi: 10.1097/00004694199705000-00007. 
